\title{
ResearchGate
}

See discussions, stats, and author profiles for this publication at:

https://www.researchgate.net/publication/280805929

\section{The Value of Social: Comparing Open Student Modeling and Open Social Student Modeling}

CONFERENCE PAPER · JUNE 2015

DOI: 10.1007/978-3-319-20267-9_4

READS

82

5 AUTHORS, INCLUDING:

Peter Brusilovsky

University of Pittsburgh

387 PUBLICATIONS 12,646

CITATIONS

SEE PROFILE

Sibel Somyürek

Gazi University

15 PUBLICATIONS 45 CITATIONS

SEE PROFILE 


\title{
The Value of Social: Comparing Open Student Modeling and Open Social Student Modeling
}

\author{
Peter Brusilovsky $^{1(\bowtie)}$, Sibel Somyürek ${ }^{2}$, Julio Guerra ${ }^{1}$, Roya Hosseini ${ }^{1}$, \\ and Vladimir Zadorozhny ${ }^{1}$ \\ ${ }^{1}$ School of Information Sciences, University of Pittsburgh, Pittsburgh, USA \\ \{peterb, jdg60, roh38\} apitt.edu, vladimirasis.pitt.edu \\ ${ }^{2}$ Department of Computer Education and Instructional Technologies, \\ Gazi University, Ankara, Turkey \\ ssomyurek@gazi.edu.tr
}

\begin{abstract}
Open Student Modeling (OSM) is a popular technology that makes traditionally hidden student models available to the learners for exploration. OSM is known for its ability to increase student engagement, motivation, and knowledge reflection. A recent extension of OSM known as Open Social Student Modeling (OSSM) attempts to enhance cognitive aspects of OSM with social aspects by allowing students to explore models of peer students or the whole class. In this paper, we introduce MasteryGrids, a scalable OSSM interface and report the results of a large-scale classroom study that explored the value of adding social dimension to OSM. The results of the study reveal a remarkable engaging potential of OSSM as well as its impact on learning effectiveness and user attitude.
\end{abstract}

Keywords: Open student modeling $\cdot$ Open social student modeling $\cdot$ Social visualization

\section{Introduction}

Open student modeling (known also as open learner modeling) is a popular technology in the field of adaptive educational systems. In the majority of adaptive educational systems, student models, which represent the current state of student domain knowledge, are hidden and used exclusively to support various kinds of learning personalization. In contrast, systems with open student modeling (OSM) provide an interface for the user to view (and even edit) the content of their models [4]. More than a decade of research on OSM demonstrated a number of valuable features of this technology such as support of self-reflection and selfregulated learning, better personalization transparency, and increased user motivation $[4 ; 5 ; 8 ; 11]$.

Much less explored is a recent extension of OSM known as open social student modeling (OSSM). The idea of OSSM is to enhance the cognitive aspects of OSM with social aspects by allowing students to explore each other models or cumulative model of the class. While several pioneer project demonstrated the feasibility of this 
approach and reported first positive results [3; 7], the value of adding social dimension to the classic OSM is still not demonstrated reliably. In this paper we present our most recent attempts to explore the added value of OSSM. We present an implementation of OSSM in an open source system MasteryGrids and report the results of a larger-scale classroom study comparing an OSSM version of MasteryGrids with a baseline OSM version. The results of the study indicate a number of benefits that could be offered by OSSM.

\section{Background: Open Student Models}

An open student model was originally suggested an innovation in the area of personalized learning systems. While in traditional personalized systems, student models were hidden "under the hood" and used to make education process personalized, the pioneers of open student modeling argued that the ability to view and modify the state of their knowledge could be beneficial for the students. A typical OSM displays the modeled state of student knowledge, although the examples of models displaying interests [1] or learning styles [12] are also known. A common way to display a state of knowledge is a set of skillometers that show the mastered subset of expert knowledge $[11 ; 15 ; 16]$ or the probability that a learner knows a concept [6]. More complex OSM could display misconceptions, the size of topics, and other factors [4].

The idea to make open student modeling social has been originally suggested and explored by Bull [3; 4]. The idea of OSSM is to enhance its cognitive aspects with social aspects by allowing students to explore each other models or cumulative model of the class. In our earlier work, we explored several approaches to combine open social student modeling with adaptive navigation support in an adaptive system for Java programming. Our preliminary single-classroom studies demonstrated that open social student modeling increase learner motivation to learn and enhance the impact of adaptive navigation support [7;9]. The study presented in this paper makes a closer look of the effects of the open student model with and without social comparison features and differs from earlier studies by its more formal nature, larger scale and different domain (SQL).

\section{MasteryGrids, an Open Social Student Modeling Interface}

To evaluate the effectiveness of OSSM we used MasteryGrids, an open source OSSM interface developed by our group [10]. MasteryGrids uses parallel social visualization approach pioneered in an earlier system Progressor+ [7]. The idea of MasteryGrids is to show progress of student knowledge over multiple kinds of educational content organized by topics, allow to compare personal progress with the progress of the class, and provide direct access to the content. Figure 1 shows MasteryGrids interface displaying a sequence of topics of a Database Management course. The first row of the grid presents the topic-by-topic knowledge progress of the current student using green colors of different density (the darker is the color, the higher is the progress). The third row shows the aggregated topic-by-topic progress of the whole class using blue colors of different density. The second row presents a color-difference comparison between the student progress and the class progress. Green color shows topics where the student 
estimated knowledge is higher than the class, blue color show the topics where the class is ahead, and the color density shows the magnitude of the difference. By clicking on any topic cell, the student can access learning content associated with the topic. For example, in Figure 1, the student has clicked the topic SELECT-FROM-WHERE and the system displays a bubble with showing content of two kinds: problems (called quizzes) and examples. Depending on the row clicked, the colors of the content items can represent individual progress, class progress, or difference using the color schemas explained before. In Figure 1, comparison colors are displayed since the student accessed content bubble by clicking on the middle comparison row. In addition to displaying the overall class progress, MasteryGrids can display anonymized ranked list of individual student models as shown in Figure 2. To save time and space, this list has to be requested by clicking "Load the rest of learners" button. The position of the current student in the list is shown in green.

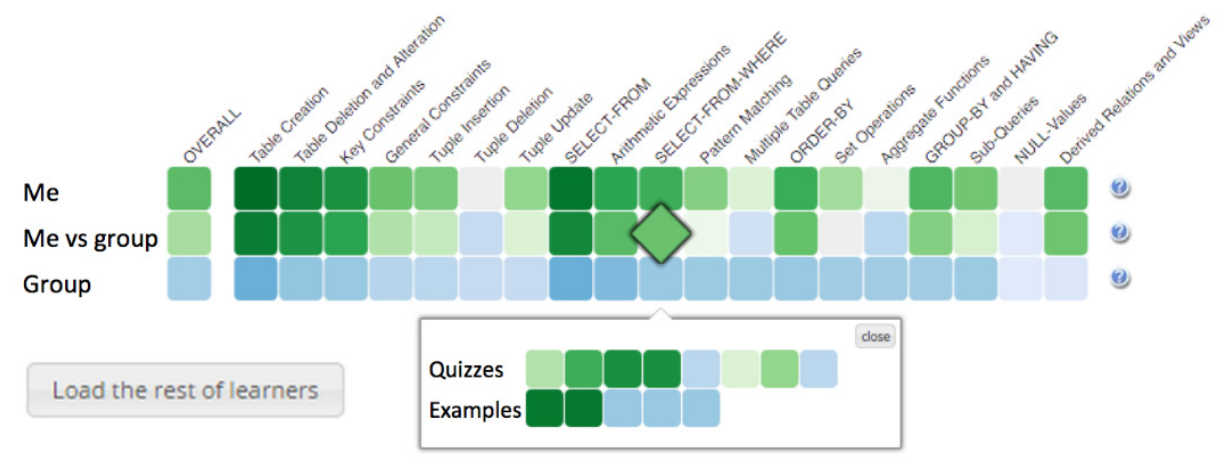

Fig. 1. MasteryGrid interface with social features activated

\section{The Study}

To assess the added impact of social features in OSM context and its implementation in Mastery Grids system, we focused on the following research questions:

1. How the addition of social dimension to OSM impacts system usage?

2. How the addition of social dimension to OSM impacts educational effectiveness?

3. How the addition of social dimension to OSM impacts learning outcomes?

4. How do students evaluate systems with and without social component?

\subsection{Study Design}

To answer these research questions we ran a classroom study where we compared two different versions of MasteryGrids, one nicknamed OSM+Social contains both the OSM and OSSM features (as shown in Figures 1 and 2), and another, nicknamed $O S M$ with OSM features only, i.e. only showing first row of the grid in Figure 1 and no access to peer list. The study was performed in a master-level Database Management course at the School of Information Sciences, University of Pittsburgh, during the term of Fall 2014. The class was divided into two comparable sections 
taught by the same instructor using the same lecture material. Sections had different class meeting times. One section was assigned to work with the OSM version of the system and another section with the OSM+Social version. Both versions provided access to the same educational content. Progress visualization in the OSM+Social group was based on the progress of this group alone.

Students in the class (you are 9th out of 50)

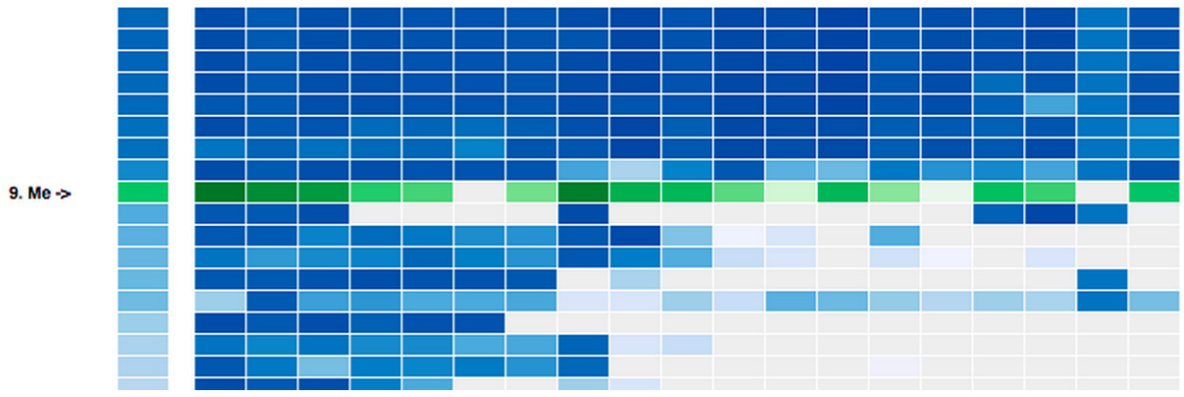

Fig. 2. Sorted list of peers. The current student can find herself, but no names are shown.

MasteryGrids was introduced to both sections in the 3rd week of the course right before the start of SQL part supported by the system. The students were informed about the study and received a quick introduction to the MasteryGrids interface used in the group and the learning content available in the system. Then students were also administered a pretest to check their SQL knowledge. Pretest included ten questions that require writing SQL statements. After the introduction, each student received e-mails with a link to access the system, individual login and password. The use of the system was not mandatory in the course, however, to motivate students to try the system, one extra credit point was offered to students who solve at least 10 problems in the system. All user interaction with the system was logged. At the end of 11th week of the course, the participants took a posttest and filled in a questionnaire about usefulness and usability of the system.

\subsection{Participants}

The total number of students in two course section was 103, however, 14 students never logged in and were excluded from the study. Out of remaining 89 students, 47 $(52.8 \%)$ worked with OSM interface and 42 (47.2\%) full OSSM (OSM+Social) interface. The descriptive statistics of experiment groups and students' gender are given in Table 1.

Table 1. The descriptive statistics of OSM+Social/OSM groups and students' gender

\begin{tabular}{ccc|cc}
\hline Systems/ & \multicolumn{2}{c|}{ OSM+Social } & \multicolumn{2}{c}{ OSM } \\
\cline { 2 - 5 } Gender & $\mathrm{f}$ & $\%$ & $\mathrm{f}$ & $\%$ \\
\hline Female & 26 & 55.3 & 21 & 50 \\
Male & 21 & 44.7 & 21 & 50 \\
\hline Total & 47 & 100 & 42 & 100 \\
\hline
\end{tabular}




\subsection{Log Data Collection}

MasteryGrids allows students to access 2 types of content: parameterized problems (referred for now on as problems) and examples. We used a set of SQL problems and examples developed for an earlier system Database Exploratorium [2]. Each problem asks the student to write a SQL statement to retrieve a subset of data from a predefined database. Problems are parameterized, which means that they are generated from a template in slightly different way (and with different correct answers) each time. As a result, students can attempt the same problem several times. Examples present various SQL statements with explanations for each line. All explanations are originally hidden; the student can explore line explanations one by one by clicking lines of interest, allowing the system to keep track of which line has been viewed. All student activity with the system (accessing topics and learning content through the MasteryGrid interface, every attempt to solve problems, every example line viewed) is logged by the system.

\section{$5 \quad$ Results}

In accordance with the research questions introduced above, the independent variable of the study is the type of interface used by the group (OSM or OSM+Social) and the dependent variables of the study are system usage, instructional effectiveness, educational impact, and students' opinions about systems usability and usefulness. We analyze these aspects in the following sections.

\subsection{Student Engagement}

In our past work, we observed that the use of open student models increases the number of students who was motivated to work with non-mandatory learning content [8]. To investigate whether OSSM is better or worse than OSM in this respect, we compared the fraction of students engaged to work with OSM and OSM+Social group at six different levels. In total there were 42 students in the OSM group and 47 in the OSM+Social group who logged in the system at least once, i.e. had a chance to see the system and to make an informed decision whether to use the system or not. In Figure 3(a) we use this starting number as $100 \%$ to compare the percentage of students in each group who had at least one, more than 10, 20, 30, 40, and 50 attempts on problems. As we can see, the OSM+Social group has much higher student engagement on all levels. The most remarkable difference is observed at the early engagement stages. The social version was apparently looking much more interesting to the students: almost $70 \%$ decided to explore the system further attempting at least one question. In contrast, traditional OSM lost more than $70 \%$ of its users right after their first login - less than $30 \%$ of them decided to move on by at least trying one question. At the level of $30+$ questions that we could consider as a serious engagement with the system, the OSM+Social group still retained more than $50 \%$ of its original users while OSM engagement was below $20 \%$. 


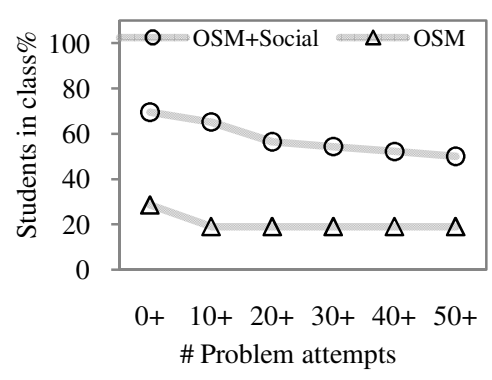

(a)

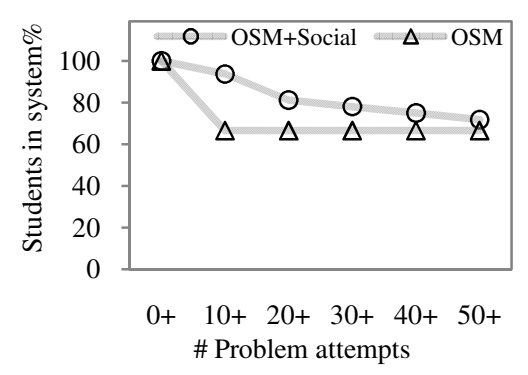

(b)

Fig. 3. Percentage of active students with different number of problem attempts in the OSM and OSM+Social group. The levels in the $\mathrm{x}$-axis represent having at least one attempt, more than $10,20,30,40$, and 50 attempts.

It could be argued that students were not able to understand the value of the system until they tried at least one problem, so using the number of students who logged in as $100 \%$ is unfair. Figure 3(b) provides an alternative look at the student engagement treating the number of students who attempted at least one problem as $100 \%$ in each group. Still, we see that OSM group is loosing students at a higher rate than the OSM+Social group even with this adjustment. These observations demonstrate that the OSM+Social group was much more successful than the OSM group in engaging and retaining students.

\subsection{System Usage}

To further compare the ability of two system versions to engage students, we examined the variables in Table 2. Since the data could not be assumed to be normally distributed, Mann Whitney $\mathrm{U}$, a nonparametric statistical test was used to compare system usage between OSM $(\mathrm{N}=42)$ and OSM+Social $(\mathrm{N}=47)$ interface groups. The Table 2 shows the results of Mann Whitney U test.

Table 2. The results of Mann Whitney U test about system usage. ${ }^{*}$ Significant result $(\mathrm{p}<0.05)$ ** Significant result $(\mathrm{p}<0.01)$.

\begin{tabular}{lc|cc}
\hline Variable & OSM & OSM+Social & U \\
\cline { 2 - 3 } & Mean & Mean & \\
\hline Sessions & 3.93 & 6.26 & $685.500^{* *}$ \\
Topics coverage & $19.0 \%$ & $56.4 \%$ & $567.500^{* *}$ \\
Total attempts to problems & 25.86 & 97.62 & $548.500^{* *}$ \\
Correct attempts to problems & 14.62 & 60.28 & $548.000^{* *}$ \\
Distinct problems attempted & 7.71 & 23.51 & $549.000^{* *}$ \\
Distinct problems attempted correctly & 7.52 & 23.11 & $545.000^{* *}$ \\
Distinct examples viewed & 18.19 & 38.55 & $611.500^{* *}$ \\
Views to example lines & 91.60 & 209.40 & $609.000^{* *}$ \\
MG loads & 5.05 & 9.83 & $618.500^{* *}$ \\
MG clicks on topic cells & 24.17 & 61.36 & $638.500^{* *}$ \\
MG click on content cells & 46.17 & 119.19 & $577.500^{* *}$ \\
MG difficulty feedback answers & 6.83 & 14.68 & $599.500^{* *}$ \\
Total time in the system & 5145.34 & 9276.58 & $667.000^{* *}$ \\
Time in problems & 911.86 & 2727.38 & $582.000^{* *}$ \\
Time in MG (navigation) & 2260.10 & 4085.31 & $625.000^{* *}$ \\
\hline
\end{tabular}


The results indicated that students who used the OSM+Social interface were significantly more engaged with the system, i.e., logged in more frequently, covered more topics, answered more problems, tried to solve and correctly answered more problems, explored more examples and example lines, worked more extensively with MasteryGrids interface, and spent more time working with the system.

\subsection{Effectiveness}

As it can be seen in the following table, time per line, time per example and time per activity scores of students in who studied with OSM+Social interface are significantly lower than the other group. To avoid impact of users on early stage of system experience who might work less efficiently, we excluded from calculations users who explored less than 5 example (first two lines), solved less than 5 problems $\left(3^{\text {rd }}\right.$ line) and those explored less than 5 examples or solved less than 5 problems ( $4^{\text {th }}$ line).

Table 3. The results of Mann Whitney U test about productivity scores. * Significant result $(\mathrm{p}<0.05){ }^{* *}$ Significant result $(\mathrm{p}<0.01)$.

\begin{tabular}{lc|cc}
\hline Variable & OSM & OSM+Social & U \\
\cline { 2 - 3 } & Mean & Mean & \\
\hline Time per line & 22.93 & 11.61 & $570.000^{* *}$ \\
Time per example & 97.74 & 58.54 & $508.000^{*}$ \\
Time per problem & 37.96 & 29.72 & 242.000 \\
Time per activity & 47.92 & 34.33 & $277.000^{*}$ \\
\hline
\end{tabular}

This result shows students who used OSM+Social interface worked more efficiently than OSM group. This might be the result of social navigation support provided by OSSM interface that brought students to the right content at the right time. It might be also caused by students' attempts to move ahead of their classmates in the visualization. Further research is needed to investigate the nature of this effect.

Instructional effectiveness could be measured more reliably using an approach that takes into account both time and success such as the computational procedure developed by Paas and Van Merrienboer's [13; 14]. To find out instructional effectiveness following this approach, the performance (correctly answered SQL problems) was combined with time (total time that spend to answer SQL problems). The raw scores of performance and time were firstly translated to $\mathrm{Z}$ scores and were plotted in a Cartesian Plane. Then relative instructional effectiveness is computed as the distance between the point $(\mathrm{z}(\mathrm{P}), \mathrm{z}(\mathrm{t}))$ to the line of zero effectiveness $(\mathrm{E}=0)$.

To compare instructional effectiveness of students, only the data belong to students who solved at least 5 distinct problems in both two groups are used $(\mathrm{N}=44)$. According to results of Mann Whitney $U$ test, instructional effectiveness scores of students who studied with OSM+Social interface are significantly higher $(\mathrm{N}=32$, mean $=0.22$, mean $r a n k=24.88$ ) than the scores of students who studied with the OSM interface $(\mathrm{N}=12$, mean $=0.03$, mean rank=16.17). 


\subsection{Learning Impact}

To see the effect of social interface on students' learning, we measured the normalized learning gain of students using their scores on the pretest and posttest (ngain=(posttestpretest)/(maxscore-pretest)). For this analysis, we considered students with at least 5 attempts on problems who answered both pretest and posttest. After this filtering, there were 12 students in the OSM group and 30 students in the OSM+Social group. While the mean learning gain of students in $\mathrm{OSM}+$ Social group $(\mathrm{M}=0.47, \mathrm{SD}=0.11)$ was higher than in the OSM grop $(\mathrm{M}=0.41, \mathrm{SD}=0.17)$ the different was not significant $(\mathrm{p}=.173)$. It is, however, quite common than innovative technology most significantly affect weaker students. To check whether it is true in our case, we measured the learning gain for weak and strong students separately. If a student achieved less than $25 \%$ of the score in the pretest (is was $1 / 2$ of the top pretest score or $50 \%$ achieved by our students), we classified the student into the weak group, otherwise strong group ${ }^{1}$. Table 4 reports summary of learning gain for weak and strong students inside each group. As we found, the mean learning gain was higher for both weak and strong students in the OSM+Social group compared to the OSM group and the difference was significant for weak students $(\mathrm{p}=.033)$.

Table 4. Mean $\pm \mathrm{SD}$ of normalized learning gain of weak and strong students in the OSM and OSM+Social group

\begin{tabular}{cccc|ccc}
\hline \multicolumn{3}{c|}{ Weak $(\mathrm{n}=35)$} & \multicolumn{3}{c}{ Strong $(\mathrm{n}=7)$} \\
\hline \multirow{2}{*}{ ngain } & OSM $(\mathrm{n}=9)$ & OSM+Social $(\mathrm{n}=26)$ & $\mathrm{p}$-value & OSM(n=3) & OSM+Social $(\mathrm{n}=4)$ & $\mathrm{p}$-value \\
\cline { 2 - 7 } & $0.35 \pm 0.15$ & $0.45 \pm 0.1$ & .033 & $0.57 \pm 0.14$ & $0.6 \pm 0.13$ & .824 \\
\hline
\end{tabular}

We also examined the association between number of activity attempts in each group and the final grades of the students in the class. We fitted a mixed model with group (G), number of attempts on problems (NP), examples (NE), and example lines (NL) as the fixed effects and the final grade as the response variable. We found that the group $(\mathrm{G})$, number of examples (NE), and lines (NL) are not significant predictor of the final grade, while number of attempted problems (NP) significantly predicts the final grade. We obtained the coefficient of 0.09 for NP meaning that attempting 1 problem in the system was associated with an increase of 0.09 in the final grade ranging from 0 to $100(\mathrm{SE}=0.04, \mathrm{p}=.017)$. In other words, attempting 100 problems will increase the final grade by 9 . This implies that in both groups, more attempts on problems may help gaining better grade in the final exam. This shows that better ability of OSSM interface to engage students in problem solving is important.

\subsection{Questionnaire Analysis}

A total of 81 students (42 in OSM+Social group, 39 in OSM group) answered the questionnaire about usability and usefulness of MasteryGrids. To focus on more informed feedback, we discarded all students who have used the system less than

1 Using other thresholds such as $10 \%$ (median) and $20 \%$ did not change the results substantially. 
300 seconds, keeping 53 students' responses for further analysis: 32 in OSM+Social group (18 females, 14 males), and 21 in OSM group (10 females, 11 males).

Table 5 presents the questions in each part of the questionnaire with Mean and Standard Error of the Mean for each group. Values range from 1 (strongly disagree) to 5 (strongly agree). Part 1 was answered by all 53 students; part 2 was answered only by students in OSM group; part 3 was answered by students in OSM+Social group, from which we discarded all answers which value (3) was tagged as "Did not notice", keeping 17 to 26 responses (some students did not answer all of the questions). In the next paragraphs we refer to the questions in Table 5 as PXQY where $\mathrm{X}$ represents the part $(1,2,3)$ and $Y$ the question number.

The general usability and usefulness of the system in Part 1 are evaluated positively, with values generally above 3.5 and many of them above 4 in OSM+Social. There is a clear tendency of more positive answers in OSM+Social group, although the only significant difference observed between groups is in P1Q3: students in OSM+Social group $(\mathrm{N}=31)$ stated to be more motivated than students on the OSM group $(\mathrm{N}=21)$ by the self-progress features in MasteryGrids, Mann-Whitney $\mathrm{U}=225, \mathrm{p}=.026$ two-tailed. We followed this analysis contrasting the response of OSM+Social group in P1Q3 with the similar question about OSSM features, P3Q10, and we found a significant difference $\mathrm{p}=.031$ (using Wilcoxon Signed Rank test): while log data shows that students in the OSM+Social group used the system much more than the students in OSM group, they were also more eager to trace it to the ability of seeing their own progress.

To examine the impact of in-system experience, we cluster students into usage groups, low $(\mathrm{N}=26)$ and high $(\mathrm{N}=27)$ using a two steps clustering over standardize values of the system usage variables number of distinct problems attempted, number of distinct examples viewed, number of clicks in topics cells and number of clicks in content cells (problems and examples). We expected that students who used the system more will evaluate it higher, as it frequently happens with complicated systems, but we did not find any significant difference here. We hypothesized that the system was sufficiently simple and usable to be mastered even the low group.

Part 2, answered by OSM group, presents questions about perceived value of social features. We compared these questions with similar questions in Part 3, answered by the other group (OSM+Social) wondering if, in general, students value more the social features when they experience them or vice versa. P2Q1 was compared to an average score in questions P3Q2, P3Q3, and P3Q5 and the difference was significant, i.e., the real value of the social features was even higher that the perceived value (OSM N=19, OSM+Social N = 15, Mann-Whitney U=80, p=.0396 two-tailed). P2Q2 was compared to P3Q10, but the difference was not significant.

Part 3, answered by the OSM+Social group, was analyzed in different forms. First we look at differences across usage clusters (as defined previously) and gender, and no significant difference was found neither in usefulness questions, nor usability questions. Then we look at possible differences between questions referring to different system features. P3Q2, P3Q3 and P3Q5 refer to group comparison feature (see Fig. 1), and P3Q6 and $\mathrm{P} 3 \mathrm{Q} 8$ refer to peer list feature. We compare these two groups of questions to see if students find more useful one or the other features. The difference was not significant. 
On usability side, students agree that the colors of the system used for comparing with others are easy to understand (P3Q4, P3Q9). This is a good result, since we had concerns about the use of colors and the difficulties to understand the comparison.

It is also interesting to contrast slightly negative score of P3Q12 and the slightly positive score of P3Q14: in general students disagree with the negative effect of social comparison, although they tend to admit that sometimes it pushes them to work just for the sake of competition. Another interesting finding is the lower scores students gave to the perspective of showing names (P3Q11). Apparently, the effect of comparison does not need to be individualized to be beneficial. We further analyze the relation between P3Q11 and P3Q13 by reversing P3Q13 and classifying answers as positive (above 3 ) or negative (below 3 ), discarding answers with value 3 . We find a significant difference (using Wilcoxon Signed-Rank test) $\mathrm{p}=.034$ : 7 out of 20 students who answer both questions think that they would like to see other names, but would not like to show their names, only 1 student would show her name but think it is not useful to see other names, and 12 students match their opinion.

Table 5. Subjective evaluation questions by part. Part 1 was answered by all students. Part 2 was answered only by students in OSM group, and Part 3 by students in OSM+Social group. Some of the questions refer to figures originally included in the questionnaire (and not reproduced here), and the references were changed to [reference], e.g. [in peer list] in question 6 and 8 in Part 3.

\begin{tabular}{|c|c|c|c|c|c|}
\hline \multirow{2}{*}{\multicolumn{2}{|c|}{ Part 1}} & \multicolumn{2}{|c|}{ OSM } & \multicolumn{2}{|c|}{ OSM+Social } \\
\hline & & $\mathrm{M}$ & SE & $\mathrm{M}$ & SE \\
\hline 1 & In general, it was useful to see my progress in Mastery Grids (MG) & 3.76 & .228 & 4.03 & .145 \\
\hline 2 & In general, I liked the interface of MG & 3.86 & .221 & 3.84 & .163 \\
\hline 3 & $\begin{array}{l}\text { Seeing my progress in the tool motivated me to work on quizzes and } \\
\text { examples }\end{array}$ & 3.52 & .214 & 4.09 & .130 \\
\hline 4 & $\begin{array}{l}\text { The interface helped me to understand how the class content is } \\
\text { organized }\end{array}$ & 3.62 & .223 & 3.81 & .176 \\
\hline 5 & The interface helped me to identify my weak points & 3.52 & .190 & 3.84 & .186 \\
\hline 6 & The interface helped me to plan my class work & 3.33 & .211 & 3.22 & .160 \\
\hline 7 & It was clear how to access questions and examples & 3.81 & .264 & 3.56 & .190 \\
\hline 8 & It was useful to see my knowledge progress for each topic [in MG] & 3.71 & .171 & 4.03 & .135 \\
\hline & $\begin{array}{l}\text { It was useful to see how I am doing with individual quizzes and } \\
\text { examples }\end{array}$ & 3.71 & .197 & 4.16 & .128 \\
\hline & $\begin{array}{l}\text { Using green colors in different intensity to show my progress was easy } \\
\text { to understand }\end{array}$ & 3.90 & .217 & 4.09 & .151 \\
\hline
\end{tabular}

\begin{tabular}{lcc} 
Part 2 (only OSM) & M & SE \\
\hline $1 \quad$ The ability to see the progress of the rest of the group will make MG more & 3.53 & .246
\end{tabular} valuable for me

2 The ability to see the progress of the rest of the group will motivate me to use $3.74 \quad .227$ MG more frequently 
Table 5. (Continued)

\begin{tabular}{|c|c|c|c|}
\hline \multicolumn{2}{|c|}{ Part 3 (Only OSM+Social) } & \multirow{2}{*}{$\frac{\mathrm{M}}{3.87}$} & \multirow{2}{*}{$\begin{array}{l}\text { SE } \\
.220\end{array}$} \\
\hline 1 & It is important for me to see the progress of the rest of the class & & \\
\hline 2 & $\begin{array}{l}\text { It was useful to see the progress of the whole class as it is represented in the } \\
\text { Group row [in MG] }\end{array}$ & 3.96 & .183 \\
\hline 3 & $\begin{array}{l}\text { It was useful to see the progress of the top students as it is represented in the } \\
\text { Group row [in MG] }\end{array}$ & 4.11 & .212 \\
\hline 4 & The comparison between the group and myself [figure] is easy to understand & 4.21 & .147 \\
\hline 5 & $\begin{array}{l}\text { It was useful to see the comparison between the selected group and myself } \\
\text { [figure] }\end{array}$ & 4.14 & .151 \\
\hline 6 & It is important for me to see the progress of individual classmates [in peer list] & 3.71 & .322 \\
\hline 7 & $\begin{array}{l}\text { In general, it is useful for me to be able to compare my progress with the progress } \\
\text { of others }\end{array}$ & 3.88 & .185 \\
\hline 8 & It is important for me to see my position in the class [in peer list] & 3.96 & .213 \\
\hline 9 & $\begin{array}{l}\text { Visualizing the progress of others using blue colors of different intensities was } \\
\text { easy to understand }\end{array}$ & 4.04 & .141 \\
\hline 10 & $\begin{array}{l}\text { Viewing my classmates' progress motivated me to work more in quizzes and } \\
\text { examples }\end{array}$ & 3.88 & .193 \\
\hline 11 & $\begin{array}{l}\text { I think it would be useful for me to know the names of individual classmates in } \\
\text { [peer list] }\end{array}$ & 2.68 & .230 \\
\hline 12 & Viewing that others were more advance than me made me want to quit using MG & 2.71 & .229 \\
\hline 13 & If names are shown, I will not like to show my name in the list to others & 4.15 & .120 \\
\hline 14 & $\begin{array}{l}\text { Sometimes I just checked quizzes and examples to catch up with others rather } \\
\text { than to learn more }\end{array}$ & 3.35 & .264 \\
\hline
\end{tabular}

\section{Discussion and Conclusion}

In this paper we presented a visual implementation of open social student modeling approach and compared it to the traditional open student model without social component in a semester-long classroom study. The results confirmed a remarkable engagement power of the OSSM: a much higher ability to engage and retain students. It also motivated students to perform significantly more work with non-mandatory learning content. These features of OSSM make it very attractive for context where motivation and retention are critical, such as modern MOOCs. In addition, social visualization enabled students in OSSM group to work more efficiently, which could be attributed to the navigation support aspect of our OSSM implementation. In our future work we plan to investigate the nature of this impact using detailed student interaction traces. The work with OSSM also positively impacted student learning significantly improving learning gain of weaker students. While this could be attributed to the increased work with the content (as shown also by the correlation between the amount of work and exam grade), it is still valuable in the case of non-mandatory educational content, which the students explore at their own will. Student answers to the administered questionnaire indicated positive attitude to both, traditional OSM features and new OSSM various features. Yet, we should acknowledge that the study confirmed the value of OSSM in one specific context - a graduate class in a large US university. The impact of the same interface might be different for other groups of students and in different countries. For example, it is not clear that the effect of OSSM will be same for considerably larger or smaller groups. We plan to investigate the impact of these factors in the future work. 


\section{References}

1. Ahn, J.-w., Brusilovsky, P., Grady, J., He, D., Syn, S.Y.: Open user profiles for adaptive news systems: help or harm? In: Proc. of the 16th international conference on World Wide Web, WWW 2007. ACM, pp. 11-20 (2007)

2. Brusilovsky, P., Sosnovsky, S., Lee, D., Yudelson, M., Zadorozhny, V., Zhou, X.: Learning SQL programming with interactive tools: from integration to personalization. ACM Transactions on Computing Education 9(4), 1-15 (2010). Article No. 19

3. Bull, S.: UMPTEEN: Named and Anonymous Learner Model Access for Instructors and Peers. International Journal of Artificial Intelligence in Education 17(3), 227-253 (2007)

4. Bull, S., Kay, J.: Student Models That Invite the Learner The SMILI:() Open Learner Modelling Framework. International Journal of AI in Education 17(2), 89-120 (2007)

5. Bull, S., Kay, J.: Open Learner Models as Drivers for Metacognitive Processes. In: Azevedo, R., Aleven, V. (eds.) International Handbook of Metacognition and Learning Technologies, pp. 349-365. Springer, Berlin (2013)

6. Corbett, A.T., Anderson, J.R.: Knowledge tracing: Modelling the acquisition of procedural knowledge. User Modeling and User-Adapted Interaction 4(4), 253-278 (1995)

7. Hsiao, I.-H., Brusilovsky, P.: Motivational Social Visualizations for Personalized E-Learning. In: Ravenscroft, A., Lindstaedt, S., Kloos, C.D., Hernández-Leo, D. (eds.) EC-TEL 2012. LNCS, vol. 7563, pp. 153-165. Springer, Heidelberg (2012)

8. Hsiao, I.-H., Sosnovsky, S., Brusilovsky, P.: Guiding students to the right questions: adaptive navigation support in an E-Learning system for Java programming. Journal of Computer Assisted Learning 26(4), 270-283 (2010)

9. Hsiao, I.H., Bakalov, F., Brusilovsky, P., König-Ries, B.: Progressor: social navigation support through open social student modeling. New Review of Hypermedia and Multimedia 19(2), 112-131 (2013)

10. Loboda, T.D., Guerra, J., Hosseini, R., Brusilovsky, P.: Mastery Grids: An Open Source Social Educational Progress Visualization. In: de Freitas, S., Rensing, C., Ley, T., Muñoz-Merino, P.J. (eds.) EC-TEL 2014. LNCS, vol. 8719, pp. 235-248. Springer, Heidelberg (2014)

11. Mitrovic, A., Martin, B.: Evaluating the Effect of Open Student Models on SelfAssessment. International Journal of AI in Education 17(2), 121-144 (2007)

12. O'Keeffe, I., Brady, A., Conlan, O., Wade, V.: Just-in-time Generation of Pedagogically Sound, Context Sensitive Personalized Learning Experiences. International Journal on E-Learning 5(1), 113-127 (2006)

13. Paas, F., van Merriënboer, J.J.G.: The efficiency of instructional conditions: An approach to combine mental effort and performance measures. Human Factors 35, 737-743 (1993)

14. Paas, F., van Merriënboer, J.J.G.: Instructional control of cognitive load in the training of complex cognitive tasks. Educational Psychology Review 35(6), 51-71 (1994)

15. Papanikolaou, K.A., Grigoriadou, M., Kornilakis, H., Magoulas, G.D.: Personalising the interaction in a Web-based Educational Hypermedia System: the case of INSPIRE. User Modeling and User Adapted Interaction 13(3), 213-267 (2003)

16. Weber, G., Brusilovsky, P.: ELM-ART: An adaptive versatile system for Web-based instruction. International Journal of Artificial Intelligence in Education 12(4), 351-384 (2001) 